\title{
Non-breaking and breaking solitary wave run-up
}

\author{
By YING LI ${ }^{1}$ AND FREDRIC RAICHLEN ${ }^{2}$ \\ ${ }^{1}$ Oracle Corporation, Redwood Shores, CA 94065, USA \\ ${ }^{2}$ California Institute of Technology, Pasadena, CA 91125, USA
}

(Received 5 December 2000 and in revised form 12 October 2001)

The run-up of non-breaking and breaking solitary waves on a uniform plane beach connected to a constant-depth wave tank was investigated experimentally and numerically. If only the general characteristics of the run-up process and the maximum run-up are of interest, for the case of a breaking wave the post-breaking condition can be simplified and represented as a propagating bore. A numerical model using this bore structure to treat the process of wave breaking and subsequent shoreward propagation was developed. The nonlinear shallow water equations (NLSW) were solved using the weighted essentially non-oscillatory (WENO) shock capturing scheme employed in gas dynamics. Wave breaking and post-breaking propagation are handled automatically by this scheme and ad hoc terms are not required. A computational domain mapping technique was used to model the shoreline movement. This numerical scheme was found to provide a relatively simple and reasonably good prediction of various aspects of the run-up process. The energy dissipation associated with wave breaking of solitary wave run-up (excluding the effects of bottom friction) was also estimated using the results from the numerical model.

\section{Introduction}

Tsunamis are sea waves generated by underwater earthquakes or landslides. As a tsunami propagates shoreward it undergoes changes caused by the offshore bathymetry and can increase significantly in height and break near the shoreline, travelling inland with the potential for causing large property damage and loss of life. For example, a recent event in Papua New Guinea in July 1998 resulted in widespread destruction and more than 2000 deaths. From eye witness accounts it appears that the waves, some about $4 \mathrm{~m}$ to $15 \mathrm{~m}$ high at the shoreline, were breaking (see Kawata et al. 1999). Since most of the damage associated with tsunamis is related to their run-up at the shoreline, understanding and being able to predict the run-up associated with such long incident breaking waves is an important aspect of a seismic sea wave mitigation effort.

Analytical models have been developed for non-breaking wave run-up, e.g. see Synolakis (1987). However, due to the complex nature of the fluid motion during the process of wave breaking, by necessity any models of the run-up of breaking long waves must be numerical. For example, an early study by Brennen \& Whitney (1970) used the inviscid dynamical equations of motion in Lagrangian coordinates to investigate the run-up of periodic waves; their calculations were reasonable for non-breaking waves, but the computations became unstable when the wave broke and were terminated.

The nonlinear shallow water wave equations (NLSW) have been employed widely 
to model non-breaking wave propagation and run-up. The analogy between a shallow water breaking wave and a shock wave in gas dynamics suggests that if provision is made in the numerical model of the NLSW to account for the energy dissipation associated with wave breaking and post-breaking propagation, these equations may be used also to simulate the run-up of breaking waves. Hibbert \& Peregrine (1979) solved the NLSW equations in conservative form using the Lax-Wendroff scheme, and applied the scheme to calculate the evolution and run-up of a uniform bore on a slope. The moving shoreline was treated by adding new grid points during runup, and, if necessary, subtracting the points that were not covered by water during run-down. Although this scheme is not robust, Hibbert \& Peregrine (1979) gave the first quantitative and realistic solution of the behaviour of a uniform bore during the run-up process.

Titov \& Synolakis (1995) solved the characteristic form of the NLSW equations using finite difference methods and used it to model the propagation and runup of solitary waves which break on a plane slope. The characteristic equations were solved using the Godunov scheme to avoid numerical instabilities. The moving shoreline was treated the same as that of Hibbert \& Peregrine (1979) by adding and subtracting grid points according to the shoreline position, except that the boundary conditions imposed on the shoreline were modified, in an attempt to avoid stability problems. In that regard it was assumed that the slope of the water surface was zero at the shoreline. The wave evolution and maximum run-up for non-breaking and breaking solitary waves were computed and compared with experimental results with reasonable agreement. However, in their simulations, oscillations in the results occurred near the breaking point.

Zhang (1996) showed that the zero-slope boundary condition used by Titov \& Synolakis (1995) can be improved, and he developed a finite difference scheme for the shallow water wave equations using the Lax-Wendroff scheme to investigate non-breaking solitary wave run-up. The run-up was modelled by remapping the grid points at the surface according to the instantaneous shoreline position. This computing domain mapping technique proposed by Zhang (1996) appears to treat the shoreline movement well and will be used in the numerical scheme presented herein.

Dodd (1998) investigated wave run-up, overtopping and regeneration by solving the NLSW equations using the Roe-type Riemann solver developed in gas dynamics to track shock waves. An energy dissipative term representing bottom friction was included in the model. In the scheme, a minimum local depth $d_{\min }$ was defined to treat the moving shoreline. When the water depth in the cell is less than $d_{\min }$, the cell was considered 'dry', otherwise, the cell was occupied by water ('wet'). The shoreline was defined as the line of separation between the 'dry' and the 'wet' cells. Dodd (1998) conducted simulations of wave propagation and overtopping including random waves and compared them to experimental results, and good agreement was found.

In summary, the models utilizing the NLSW equations, although having limitations such as failing to provide depthwise variations in velocity and omitting frequency dispersive effects, appear to be able to model important aspects of the wave breaking process. The additional ability of the NLSW equations to model the general characteristics of the run-up of these breaking solitary waves is considered here.

Other numerical models such as Boussinesq-type models have been used to simulate wave breaking and run-up. Up to the point of breaking these equations can represent the nonlinear effects and dispersive effects to a high degree of accuracy. However, a special 'breaking' term has to be included in the momentum conservation equation 
to model the dissipation associated with wave breaking. The term must incorporate coefficients that need to be calibrated by field or experimental data, which limits their application. Zelt \& Raichlen (1990) developed a Lagrangian representation of the Boussinesq equations and used a finite-element model to investigate the runup of both non-breaking and breaking waves on a plane beach. Wave breaking was parameterized with an artificial viscosity term in the momentum equation, and the bottom friction was also modelled as a term quadratic in the horizontal water velocity. When calibrated with laboratory data, the model of Zelt (1991) could provide reasonable predictions of the wave run-up process. (In addition, Zelt \& Raichlen 1991 using this model studied the landward inundation of non-breaking solitary waves that propagate up a non-planar slope.)

Numerical solutions of the Laplace equations and the Navier-Stokes equations also have been used in wave run-up investigations as computational power has increased and algorithms used to solve complex systems have been developed. Grilli, Svendsen \& Subramanya (1997) solved a fully nonlinear potential flow model employing boundary element techniques (BEM), and used it to calculate various characteristics of solitary wave propagation and breaking. In contrast to the depth-averaged models like the shallow water equations and the Boussinesq models, the vertical structure of the water particle kinematics could be treated numerically. Detailed wave breaking information including the shape of the plunging jet generated by the wave breaking, the celerity, and water particle velocity as well as the wave shoaling and overall wave profile were reported. However, this numerical model cannot predict maximum run-up since the computation terminates when the plunging jet strikes the free surface. Lin, Chang \& Liu (1999) developed a numerical model solving the Reynolds equations for the mean flow field and the $k-\epsilon$ equations for the turbulent kinetic energy, $k$, and the turbulence dissipation rate, $\epsilon$, and applied the model to wave breaking and run-up. The free surface locations and movement were tracked by the volume-of-fluid (VOF) method. Their numerical results agreed with experimental results in terms of the wave profile and velocities, but fail to provide jet and splash-up information.

In the present study, a numerical model, based on the nonlinear shallow water wave equations and on the weighted essentially non-oscillatory (WENO) scheme used in gas dynamics, was developed to simulate the process of wave breaking and run-up. The wave breaking process is modelled and captured automatically by the numerical scheme without introducing any ad hoc terms in the equations to suppress the numerical oscillations that are common in other numerical models. The energy dissipation associated with wave breaking, with the broken wave treated as a propagating bore, and the maximum wave run-up on a smooth slope have been studied using this numerical method. However, since the numerical model treats wave breaking only as a propagating bore, the details of the wave breaking process such as the characteristics of the plunging jet, the splash-up, etc. cannot be obtained.

\section{Mathematical formulation and numerical method}

\subsection{Mathematical formulation}

We consider the run-up of two-dimensional long waves incident upon a uniform sloping beach connected to an open ocean of constant depth as shown in figure 1. The classical nonlinear shallow water equations (NLSW) are

$$
\begin{gathered}
\eta_{t}+(u(h+\eta))_{x}=0, \\
u_{t}+u u_{x}+g \eta_{x}=0 .
\end{gathered}
$$




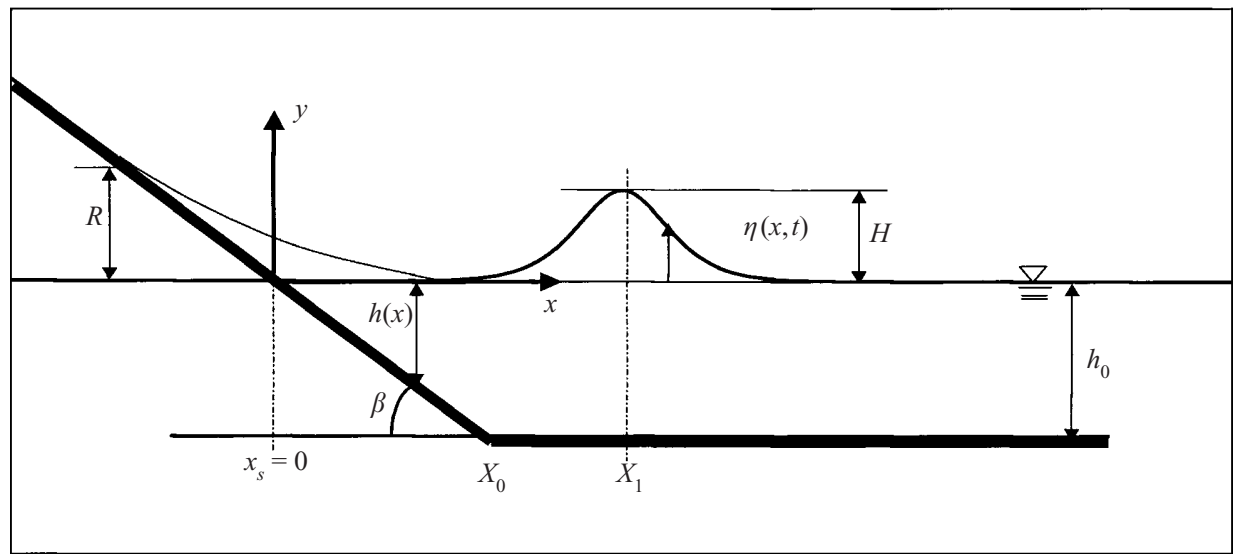

FIGURE 1. Definition sketch of the solitary wave run-up.

These equations are similar in terms of their mathematical structure to the Euler equations in gas dynamics, which can admit discontinuous solutions if written in conservative form. In gas dynamics the discontinuities are interpreted as shocks and found to be good mathematical representations of the real flow. Similarly, if we write the NLSW equations in conservative form a discontinuity in the solution is also possible, as others have found, e.g. Stoker (1957) and Titov \& Synolakis (1995) to mention only two. The discontinuity can be used as a simplified mathematical model for a breaking wave and the bore produced after wave collapse.

When written in conservative form, the shallow water equations become

$$
\begin{gathered}
(h+\eta)_{t}+(u(h+\eta))_{x}=0, \\
(u(h+\eta))_{t}+\left[(h+\eta) u^{2}+\frac{1}{2} g(h+\eta)^{2}\right]_{x}=g(h+\eta) h_{x} .
\end{gathered}
$$

Let $d=h+\eta$ and introduce the following non-dimensional variables:

$$
x^{*}=\frac{x}{h_{0}}, \quad h^{*}=\frac{h}{h_{0}}, \quad \eta^{*}=\frac{\eta}{h_{0}}, \quad t^{*}=t \sqrt{\frac{g}{h_{0}}},
$$

where $h_{0}$ is the constant depth seaward of the slope, as shown in figure 1 . The non-dimensional conservative shallow water equations are then obtained as

$$
\begin{gathered}
d_{t}+(u d)_{x}=0, \\
(d u)_{t}+\left[d u^{2}+\frac{1}{2} d^{2}\right]_{x}=d h_{x} .
\end{gathered}
$$

For convenience, the asterisk indicating non-dimensionality has been dropped in the above equations as well as in the remaining discussion.

\subsection{The numerical model and treatment of the moving shoreline}

When applying (2.6) and (2.7) to wave run-up, difficulties arise in treating the shoreline position, since the shoreline moves as the water swashes up and down the slope during the run-up process. Therefore, a special treatment is included in the numerical model to define the shoreline. We use the computational domain mapping technique developed by Zhang (1996) to model the shoreline movement. This method retains the simplicity of the Euler method, but uses a simplified Lagrangian approach for the shoreline position, and it is summarized below. 
For the computational domain $(-\Gamma, 0)$ the following transformation on $(x, t)$-plane is introduced:

$$
\begin{gathered}
x=\left(1+\frac{X(t)}{\Gamma}\right) x^{\prime}+X(t), \\
t=t^{\prime},
\end{gathered}
$$

where $X(t)$ is the shoreline position defined in the fixed coordinate system as a function of time $t, \Gamma$ is the total initial length of the computational domain, $\left(x^{\prime}, t^{\prime}\right)$ is the new computing plane. Under this transformation, in the transformed computational domain $\left(x^{\prime}, t^{\prime}\right)$, the moving shoreline position, $x=X(t)$, is always located at $x^{\prime}=0$, and the seaward boundary of the computational domain, $x=-\Gamma$, is always located at $x^{\prime}=-\Gamma$. Therefore, the length of the computational domain which extends from a location offshore of the toe of the slope to the waterline defined by the tip of the runup tongue and the number of mesh points used in the computations do not change with time during the shoreline movement process. The moving boundary problem is thus changed to a fixed boundary problem and the shoreline and its associated boundary conditions can be treated using standard techniques.

From (2.8) and (2.9) the relationship between the derivatives in the two systems are obtained as

$$
\begin{gathered}
\frac{\partial}{\partial t}=\frac{\partial}{\partial t^{\prime}}-\frac{1+x^{\prime} / \Gamma}{1+X(t) / \Gamma} U \frac{\partial}{\partial x^{\prime}}, \\
\frac{\partial}{\partial x}=\frac{1}{1+X(t) / \Gamma} \frac{\partial}{\partial x^{\prime}},
\end{gathered}
$$

where $U=\mathrm{d} X(t) / \mathrm{d} t$ is the shoreline velocity along the slope. Substituting these relations into the original equations, i.e. (2.6) and (2.7), the governing equations in the new coordinate system are obtained (the primes are dropped for convenience):

$$
\begin{gathered}
d_{t}+\left(-c_{1} U d+c_{2} u d\right)_{x}=-\frac{c_{2}}{\Gamma} U d, \\
(d u)_{t}+\left[-c_{1} U d u+c_{2} d u^{2}+\frac{1}{2} c_{2} d^{2}\right]_{x}=c_{2} d h_{x}-\frac{c_{2}}{\Gamma} U d u,
\end{gathered}
$$

where $c_{1}, c_{2}$ are defined as

$$
c_{1}=c_{1}(x, t)=\frac{1+x / \Gamma}{1+X(t) / \Gamma}, \quad c_{2}=c_{2}(t)=\frac{1}{1+X(t) / \Gamma} .
$$

Equations (2.12), (2.13) can be written in standard vector form as

$$
\boldsymbol{V}_{t}+\boldsymbol{F}_{x}=\boldsymbol{S},
$$

where $\boldsymbol{V}$ is the variable to be determined, $\boldsymbol{F}$ is the numerical flux, and $\boldsymbol{S}$ is the source term defined as,

$$
\boldsymbol{V}=\left[\begin{array}{c}
d \\
d u
\end{array}\right], \quad \boldsymbol{F}=\left[\begin{array}{c}
-c_{1} U d+c_{2} u d \\
-c_{1} U d u+c_{2} d u^{2}+\frac{1}{2} c_{2} d^{2}
\end{array}\right], \quad \boldsymbol{S}=\left[\begin{array}{c}
-c_{2} U d / \Gamma \\
c_{2} d h_{x}-c_{2} U d u / \Gamma
\end{array}\right] .
$$

Equation (2.15) is a system of hyperbolic conservation laws, and can be discretized on uniform grids by the standard finite difference method with conservative form:

$$
\frac{\boldsymbol{V}_{i}^{n+1}-\boldsymbol{V}_{i}^{n}}{\delta t}=-\frac{\boldsymbol{F}_{i+(1 / 2)}^{n}-\boldsymbol{F}_{i-(1 / 2)}^{n}}{\delta x}+\boldsymbol{S}_{i}^{n}
$$


where $\delta t$ is the computing time step, $\delta x$ is the grid size. $\boldsymbol{F}(\boldsymbol{V})$ is the numerical flux function:

$$
\boldsymbol{F}_{i+(1 / 2)}^{n}=f\left(\boldsymbol{V}_{i-r}^{n}, \boldsymbol{V}_{i-r+1}^{n}, \cdots, \boldsymbol{V}_{i+s}^{n}\right) .
$$

The quantity $f$ is a Lipschitz continuous function in all the arguments, and consistent with the physical flux $F$. These conditions ensure that if the solution to the conservative scheme (2.17) converges, it will converge to a weak solution of the original partial differential equation, i.e. (2.15). The stencil of the present numerical scheme is $(i-$ $r, i-r+1, \cdots, i+s$ ), where $r$ is the number of cells to the left of $i$ and $s$ is the number of cells to the right of $i$.

\subsection{The weighted essentially non-oscillatory (WENO) shock capturing scheme}

The differences among various numerical methods applied to the general conservation laws, (2.15), lie in the choice of the computational stencils and the numerical flux functions. Traditional numerical methods like the Lax-Wendroff method and the MacCormack method use fixed stencils for each computing point and interpolate the numerical flux function inside the stencil to obtain the flux values at the cell boundaries $i+(1 / 2)$ and $i-(1 / 2)$. These methods work well for most problems with continuous solutions. However, when applying the fixed stencil method to a problem with discontinuities within the computing domain, such as the case of breaking waves, the well-known numerical instability called the 'Gibbs phenomenon' arises where the numerical solution oscillates near the discontinuities and does not decay when the grid is refined. Various remedies have been used to eliminate or reduce these spurious oscillations. The most successful methods that have been used in the past are the artificial viscosity method and the limit flux or slope method. These methods, which introduce ad hoc 'breaking terms' to increase the numerical dissipation in the original equations or reduce the order of accuracy, are very problem dependent. Usually the coefficient in the ad hoc term which describes dissipation must be determined experimentally. In addition, since the numerical dissipation has been increased to reduce the oscillations, abrupt changes in the solution which should occur tend to be eliminated, and the numerical scheme loses accuracy.

The essentially non-oscillatory (ENO) scheme developed by Harten et al. (1987) was the first method to successfully eliminate numerical oscillations near discontinuities while retaining high-order accuracy to solve the conservative law (2.15). The difference between the ENO scheme and other methods is in how the cells are chosen and used in reconstructing the numerical flux. The basic idea of the ENO scheme is that instead of using a fixed stencil to interpolate the numerical flux function, an adaptive stencil is chosen based on the local smoothness of the solutions. The measurement of the local smoothness, the hierarchy to choose the stencil points, and the extension to higher-order schemes have been developed by Harten et al. (1987) to ensure both efficiency and accuracy for the numerical method. The ENO scheme has been used successfully in gas dynamics to simulate shock behaviour and in channel flows to simulate bores. For example, Yang \& Shu (1993) used a second-order ENO scheme to simulate bore impingement on a circular cylinder and the propagation of a bore through a channel with a contraction and an expansion.

More recently Liu, Osher \& Chan (1994) and Jiang \& Shu (1996) have developed the weighted essentially non-oscillatory scheme (WENO) based on the original ENO scheme. The WENO scheme has several improvements compared to the ENO scheme, and it can achieve a higher accuracy of the numerical flux with the same number of stencil points by exploring all the local smoothness information provided by 


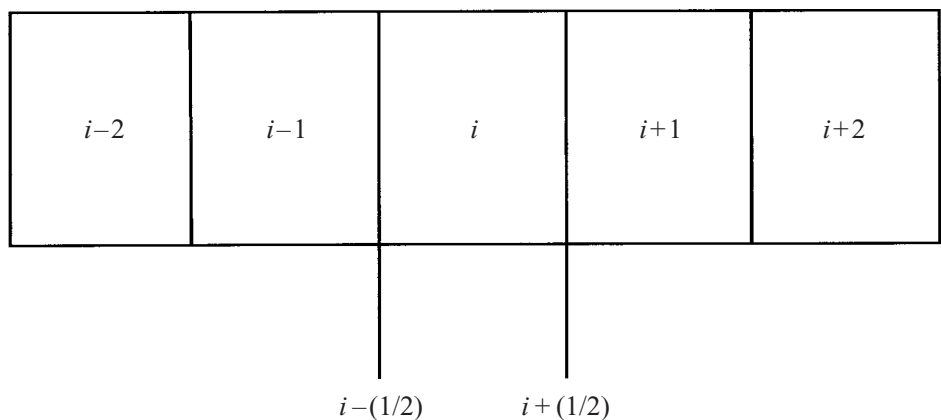

FIGURE 2. Sketch of the stencils used in the WENO scheme.

the ENO method. Applications of the WENO scheme to gas dynamics have been reported recently, e.g. Shu (1998). However applications to breaking waves and bore problems have not been reported in the literature. This study implements this scheme in simulating the breaking wave run-up process.

A detailed description of the WENO scheme can be found in Shu (1998); below is a summary of the fifth-order WENO scheme used with (2.15).

Consider the possible stencils used for calculating point $i(i-2, i-1, \ldots, i+2)$ shown in figure 2. The value of the numerical flux, $\boldsymbol{F}$, is known from (2.16). To ensure numerical stability and convergence to a physically possible solution for (2.15), LaxFriedrichs flux splitting is applied to the numerical flux:

$$
\boldsymbol{F}(\boldsymbol{V})=\boldsymbol{F}^{+}(\boldsymbol{V})+\boldsymbol{F}^{-}(\boldsymbol{V}),
$$

where

$$
\boldsymbol{F}^{ \pm}(\boldsymbol{V})=\frac{1}{2}(\boldsymbol{F}(\boldsymbol{V}) \pm \alpha \boldsymbol{V}), \quad \alpha=\max _{\boldsymbol{V}}\left|\boldsymbol{F}^{\prime}(\boldsymbol{V})\right| .
$$

(Note that $\boldsymbol{F}^{\prime}(\boldsymbol{V})$ is the derivative matrix and can be obtained easily from (2.16), and $\alpha$ is taken as the maximum over the relevant range of $u$ at each time step.)

From numerical analysis, a polynomial with third-order accuracy can be constructed from the pointwise values, $\boldsymbol{F}_{i}^{ \pm}(\boldsymbol{V})$ for the candidate stencils, and $\boldsymbol{F}_{i+(1 / 2)}^{ \pm}(\boldsymbol{V})$ in (2.17) can be obtained from this polynomial. This will give three different reconstructions by choosing different stencils that consist of three cells:

$$
F_{i+(1 / 2)}^{ \pm(r)}=\sum_{j=0}^{2} c_{r j} F_{i-r+j}^{ \pm}, \quad r=0,1,2,
$$

where $c_{r j}$ is the interpolation coefficient, which can be found in Shu (1998) and has the value

$$
c_{r j}=\sum_{m=j+1}^{k} \frac{\sum_{l=0, l \neq m}^{k} \prod_{q=0, q \neq m, l}^{k}(r-q+1)}{\prod_{l=0, l \neq m}^{k}(m-l)} .
$$

The WENO scheme gives a new approximation to the numerical flux at the boundaries 
as the combinations of these reconstructions:

$$
F_{i+(1 / 2)}^{ \pm}=\sum_{r=0}^{2} \omega_{r} F_{i+(1 / 2)}^{ \pm(r)},
$$

where $\omega_{r}$ can be obtained as

$$
\omega_{r}=\frac{\alpha_{r}}{\sum_{s=0}^{2} \alpha_{s}}, \quad \alpha_{r}=\frac{d_{r}}{\left(\epsilon+\beta_{r}\right)^{2}},
$$

and $\epsilon$ is a small number introduced to ensure that the denominator in (2.23) does not become zero; here $\epsilon=10^{-6}$ is chosen. The quantities $d_{r}, \beta_{r}$ are determined as

$$
d_{0}=\frac{3}{10}, \quad d_{1}=\frac{3}{5}, \quad d_{2}=\frac{1}{10},
$$

and

$$
\begin{gathered}
\beta_{0}=\frac{13}{12}\left(F_{i}^{ \pm}-2 F_{i+1}^{ \pm}+F_{i+2}^{ \pm}\right)^{2}+\frac{1}{4}\left(3 F_{i}^{ \pm}-4 F_{i+1}^{ \pm}+F_{i+2}^{ \pm}\right)^{2}, \\
\beta_{1}=\frac{13}{12}\left(F_{i-1}^{ \pm}-2 F_{i}^{ \pm}+F_{i+1}^{ \pm}\right)^{2}+\frac{1}{4}\left(F_{i-1}^{ \pm}-F_{i+1}^{ \pm}\right)^{2}, \\
\beta_{2}=\frac{13}{12}\left(F_{i-2}^{ \pm}-2 F_{i-1}^{ \pm}+F_{i}^{ \pm}\right)^{2}+\frac{1}{4}\left(F_{i-2}^{ \pm}-4 F_{i-1}^{ \pm}+3 F_{i}^{ \pm}\right)^{2} .
\end{gathered}
$$

By applying the above procedure to $\boldsymbol{F}_{i}^{+}(\boldsymbol{V})$ and $\boldsymbol{F}_{i}^{-}(\boldsymbol{V})$ separately for both the left boundary numerical flux $\boldsymbol{F}_{i-(1 / 2)}^{n}$ and the right boundary numerical flux $\boldsymbol{F}_{i+(1 / 2)}^{n}$ at each computing point $i$, we can obtain the total flux for the cell. From (2.17), the solutions at the new time $n+1$ can be found explicitly from the values at time level $n$. Shu (1998) indicated that the above numerical scheme is stable and is accurate up to fifth-order in smooth regions and can provide sharp discontinuities in the solutions without spurious nearby oscillations.

\subsection{Boundary conditions}

It is necessary to apply boundary conditions to the computational domain. To efficiently impose different boundary conditions, 'ghost cells' are added to the left and right boundaries. When choosing the computing stencil in the numerical scheme described above to calculate the numerical flux, only real cells are chosen during the calculation. For the totally reflective boundary conditions, e.g. for a vertical wall located at the boundary between first cell (ghost cell) and the second cell (the real cell), at the seaward boundary, the velocity of the wave at the vertical wall must be zero: $u_{1 / 2}=0$. The following boundary conditions can be derived from (2.15):

$$
\begin{gathered}
d_{0}=d_{1}, \\
(d u)_{0}=-(d u)_{1},
\end{gathered}
$$

where the cell with index 0 is the ghost cell added to the computation domain.

The characteristic approach for a hyperbolic system is used to construct the non-reflective boundary conditions at the seaward boundary, i.e. the outgoing characteristics do not carry any disturbance back to the computational domain. A detailed description of this approach can be found at Titov \& Synolakis (1995) and Zhang (1996).

For the shoreline, Zhang (1996) has proposed the following boundary conditions 
in the transformed computing domain:

$$
\begin{gathered}
h(X(t))+\eta(X(t), t)=0, \\
\frac{\mathrm{d} X(t)}{\mathrm{d} t}=U(t), \\
\frac{\mathrm{d} U}{\mathrm{~d} t}=-\eta_{x} .
\end{gathered}
$$

Equations (2.30) and (2.31) are obviously true for the shoreline position, and (2.32) is identical to (2.2). In essence these are the Lagrangian descriptions of the shoreline movement.

The Beam-Warming scheme and trapezoidal integration are used when discretizing (2.30) to (2.32) following Zhang (1996). These schemes are second-order in space and time:

$$
\begin{gathered}
U_{N}^{n+1}=U_{N}^{n}-\frac{\delta t}{2 \delta x}\left(3 \eta_{N}^{n}-4 \eta_{N-1}^{n}+\eta_{N-2}^{n}\right)+\frac{\delta t^{2}}{2 \delta x^{2}}\left(\eta_{N}^{n}-2 \eta_{N-1}^{n}+\eta_{N-2}^{n}\right), \\
X_{N}^{n+1}=X_{N}^{n}+\frac{1}{2} \delta t\left(U_{N}^{n+1}+U_{N}^{n}\right),
\end{gathered}
$$

where $N$ is the last grid index of the transformed computing domain, and always corresponds to the shoreline position.

\subsection{Test cases}

In this section the numerical scheme described in $\$ \S 2.2$ and 2.3 including the boundary conditions described in $\S 2.4$ are verified by two numerical experiments.

\subsubsection{A solitary wave propagating on a constant water depth}

This test case models a solitary wave propagating in a constant depth and is used to verify both the accuracy of the numerical scheme when solving the wave problem and the conservation laws of mass and energy in the computing domain.

We use the first-order solitary wave theory for the initial wave shape and wave velocity, i.e.

$$
\begin{gathered}
\eta=H \operatorname{sech}^{2}\left(\sqrt{\left(3 H / 4 h_{0}^{3}\right)}\left(x-X_{1}\right)\right), \\
u=\frac{c \eta}{1+\eta}, \\
c=\sqrt{g\left(H+h_{0}\right)},
\end{gathered}
$$

where $H$ is the initial relative wave height of the solitary wave, $X_{1}$ is the position of the initial wave crest in the computing domain as shown in figure 1, and $c$ is the wave celerity. It is well known that a disturbance such as a solitary wave propagating into still water of constant depth under the shallow water equations will ultimately develop a vertical leading face and break, see Stoker (1957). (This can be proved by analysing the characteristic curves for the simple wave case.) For a wave of permanent form, dispersive effects cannot be neglected for it is the balance between nonlinear effects and dispersive effects which results in a stable and permanent formed wave. To include dispersive effects in this numerical scheme, we include the dispersive term of the general Boussinesq model, see Wu (1979), into our numerical scheme. The treatment of this dispersive term is the same as that of Zhang (1996). A comparison 


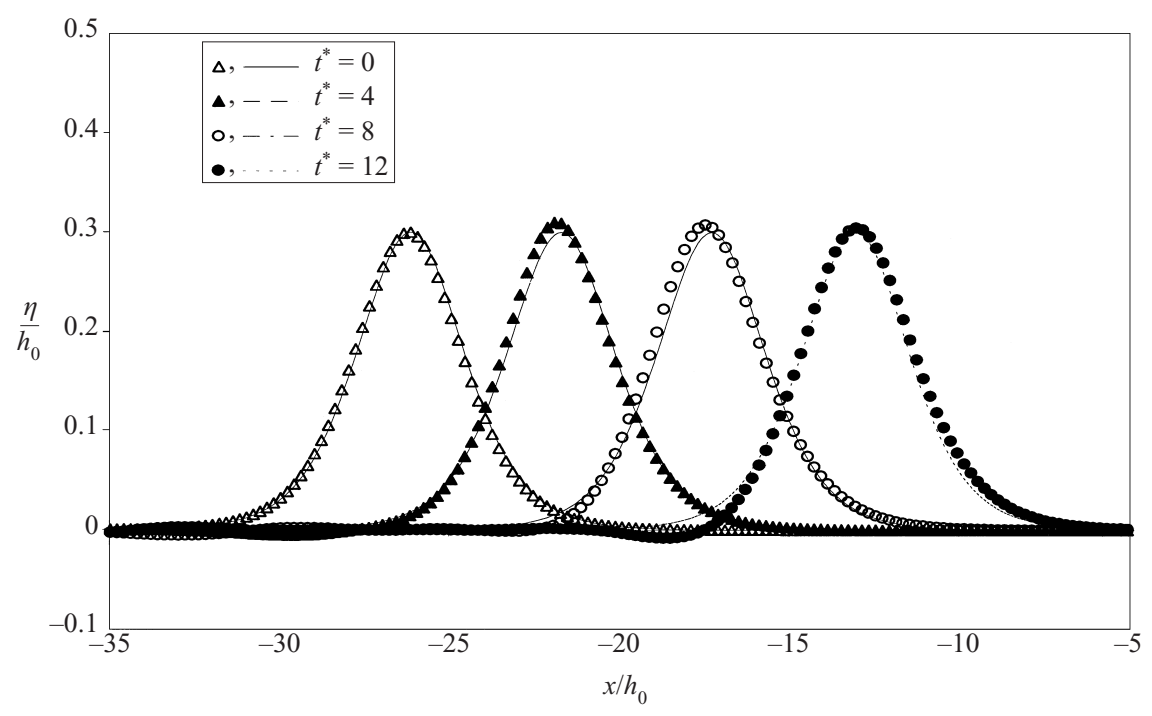

FIGURE 3. Theoretical result (lines), and numerical simulation (symbols) of propagation of a solitary wave with $H / h_{0}=0.30$ over constant water depth. Shape of the wave at different times.

between the wave shape obtained from the numerical and theoretical results is presented in figure 3. It can be seen that the solitary wave maintains its original shape as it propagates, and that the agreement between the theoretical and the numerical results is good. Indeed the amplitude of the solitary wave is approximately constant during the interval shown, with a numerical error of less than $0.1 \%$. Figure 3 suggests that the WENO scheme has a high order of accuracy in smooth regions. Note that for this case the Boussinesq term is discretized using a fixed stencil. This will cause numerical instability when simulating the wave breaking process, and hence it cannot be used in the breaking wave run-up simulation. However, as pointed out by $\mathrm{Li}$ (2000), for the wave run-up process the dispersive term will be small compared to the nonlinear term and thus it can be neglected.

The conservation of mass and energy were investigated for this numerical experiment. Mass conservation is guaranteed by the governing equation (2.3). For continuous solutions, mass conservation (2.3) and momentum conservation (2.4) are equivalent to energy conservation, thus mass and energy should be conserved for solitary wave propagation. The variation of the mass (volume) and energy in the computational domain as a function of time are presented in figure 4 . The normalized quantities are as follows:

$$
E^{*}=\frac{E}{\rho g h_{0}^{3}}, \quad V^{*}=\frac{V}{h_{0}^{2}} .
$$

We can see that the mass and energy are indeed preserved during the computation. (However, for solutions with discontinuities, the energy will not be conserved but it will decrease across the shock. This can be interpreted as the energy dissipation during the wave breaking process.)

\subsubsection{Dam-break problem}

The dam-break problem is an interesting theoretical and practical engineering problem. Various theoretical and experimental investigations have been conducted in the past to study this flow. Here we use the numerical scheme described earlier 


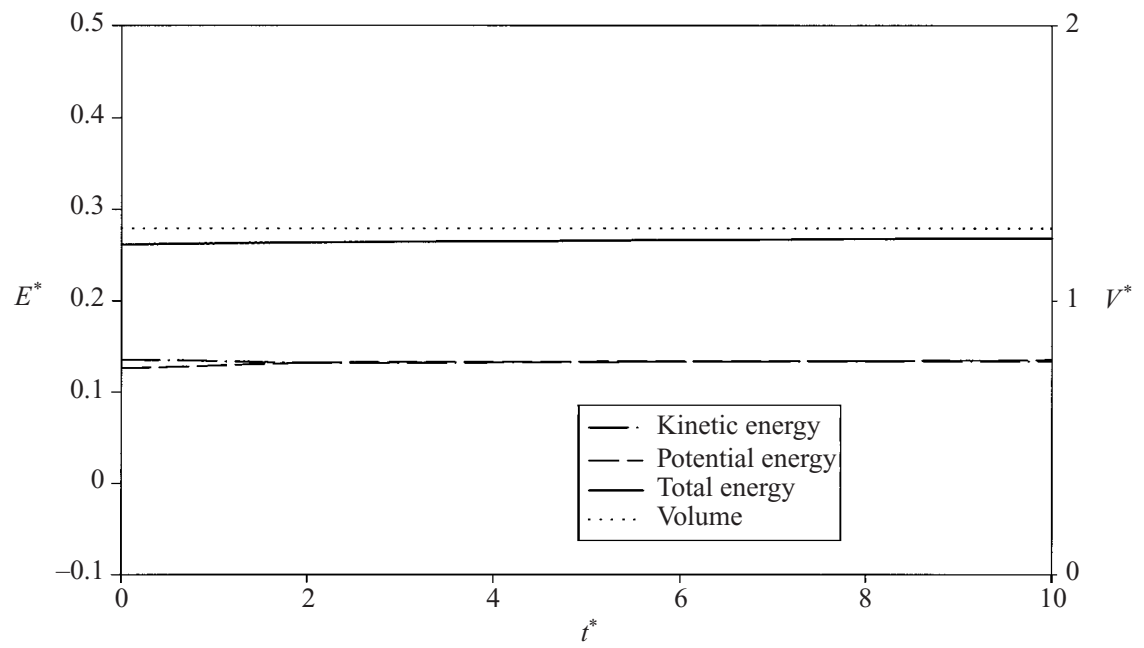

FiguRE 4. Numerical simulation of propagation of a solitary wave with $H / h_{0}=0.30$ over constant water depth. Evolution of the potential energy, kinetic energy, total energy, and volume as a function of normalized time.

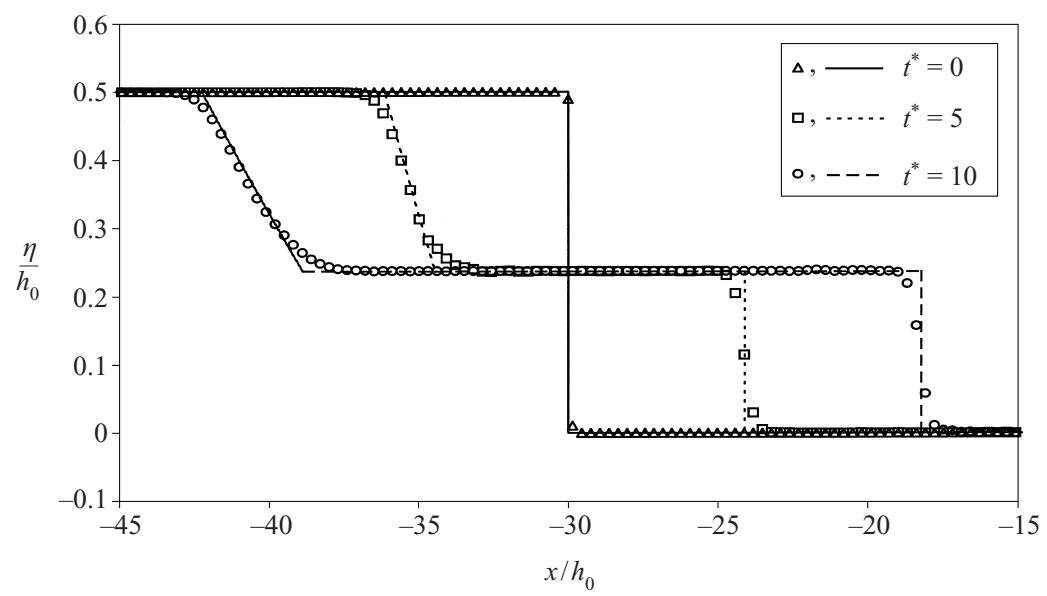

FIGURE 5. Theoretical results (lines) and numerical simulation (symbols) of dam-break flow with the ratio between upstream water depth and downstream water depth $h_{1} / h_{2}=1.5$.

to simulate the flow and compare the numerical results to the theoretical results of Stoker (1957). The initial water is still and two different water depths are separated by a thin plate: an upstream depth of $h_{1}=1.5$, and a downstream depth of $h_{2}=1.0$, as shown in figure 5. At $t^{*}=0$ the plate is removed and an expansion wave propagates upstream and a bore (discontinuity) travels downstream. A comparison between the theoretical solutions and the numerical results for the water free surface at $t^{*}=0$, $t^{*}=5.0, t^{*}=10.0$ is shown in figure 5 . We can see that the numerical results agree well with the theory even near the sharp discontinuities of the bore. It appears that the numerical scheme can reconstruct the bore within two to three cells, and neither obvious numerical dissipation nor oscillations can be observed in the solution. This suggests that the WENO scheme can indeed capture a shock (bore) without spurious oscillations while maintaining high-order accuracy in smooth shockless regions. 


\section{Experimental equipment and numerical procedures}

\subsection{Experimental setup}

A series of experiments was conducted to verify the numerical scheme which has been used for breaking solitary wave run-up. Experiments were performed in two different wave tanks: a tank $36.6 \mathrm{~m}$ long, $0.4 \mathrm{~m}$ wide, and $0.61 \mathrm{~m}$ deep located at the California Institute of Technology (CIT); and a tank $45.7 \mathrm{~m}$ long, $0.9 \mathrm{~m}$ wide and $0.9 \mathrm{~m}$ deep located at the Coastal Engineering Research Center of the US Army Corps of Engineers' Waterways Experiment Station (CERC). In both facilities, solitary waves were generated with a programmable vertical bulkhead wave generator using a method developed by Goring (1979) to generate first-order solitary waves. It has been found that waves are highly reproducible using this method: the amplitude of the wave can be reproduced within $\pm 1 \%$. The detailed laboratory setup and wave generating systems have been described in Li (2000). The laboratory run-up data of Synolakis (1986) conducted in the CIT wave tank with a beach slope of $1: 19.85$ also were used to compare with the numerical results along with our experimental results.

In the CIT tank for our studies, the 'beach' located at one end of the tank was $13.7 \mathrm{~m}$ long and composed of five panels of $0.64 \mathrm{~cm}$ thick precision aluminium plate mounted on an aluminium frame with adjustable legs; thus, the slope of the beach could be readily changed from nearly vertical to $1: 20$. The deviation of the beach from a plane surface was less than $\pm 1 \mathrm{~mm}$. The CERC wave tank had a painted plywood beach built at a fixed slope of $1: 15$ near one end.

The water surface time histories in the constant depth portion of the tank were obtained with a resistance-type wave gauge. In the CIT tank on the slope, both below and above the initial shoreline position, water surface time histories were obtained using a capacitance wave gauge consisting of a single stainless steel probe within a glass capillary tube with the tip close to the surface of the slope. This wave gauge was calibrated before and after the experiments by moving it, mounted on an instrument carriage, incrementally into deeper water with its tip always the same fixed distance above the slope. (Water surface measurements were only made in the constant depth portion of the tank in the CERC experiments and only measurements of maximum run-up were obtained there.) A high-speed video camera which can record and store a sequence of video images of an event at rates of 60 to 500 frames per second with a maximum shutter speed of $10^{-4} \mathrm{~s}$ was used to define certain water surface profiles and the run-up time history in the CIT experiments. The former were obtained with the camera mounted with its focal plane axis approximately parallel to the sidewall of the wave tank, and the latter images were obtained with the camera located above the wave tank with the axis of the focal plane approximately parallel to the sloping beach. In both cases the video camera and its associated lights were mounted on a movable instrument carriage so that successive sections of the wave and of the run-up tongue could be easily imaged. It was found that the images obtained using the high-speed video were often distorted due to the combination of the viewpoint of the camera and its optics. An image processing procedure was developed using a linear transformation from the physical coordinate system to that on the recording plane of the camera. This method of correcting for image distortion used fiducial marks on the wave tank in the calibration procedure.

The water particle velocities were measured using a back-scattering laser Doppler velocimeter with the transmitting head connected by fibre optics to the laser. The frequency range of the signal processor was $1 \mathrm{kHz}$ to $15 \mathrm{kHz}$, with a time resolution of the measurement of $2 \mathrm{~ns}$. A frequency shift of $200 \mathrm{kHz}$ between the reference beam 
and the scattering beam was sufficient to measure the water particle velocities of the solitary wave before and close to breaking as well as within the run-up tongue. Of course, air bubbles entrained during the breaking process precluded measurements immediately after breaking.

\subsection{Numerical procedures}

During the numerical simulations, a computational domain $\Gamma$ with a length corresponding to 20 constant water depths (see (2.8)) was chosen for the non-breaking solitary wave run-up and to 40 water depths for breaking solitary wave run-up on a $1: 19.85$ slope case. This includes the region of the sloping beach and a section of the constant-depth wave tank which has a length larger than twice the characteristic wavelength of incident solitary wave. The reason for choosing a large computing domain is to minimize the effect of the seaward boundary on the run-up process. The computing domain was discretized as 200 uniform grid cells after investigating the effect of the resolution on the solution for a range of values of grid cells from 100 to 300. The normalized time step in the simulation is chosen as large as possible to improve the calculation efficiency and at the same time to ensure the stability of the scheme. For non-breaking wave run-up, $\delta t=0.01$, and $\delta t=0.03$ for breaking wave run-up.

\section{Results and discussion}

In this section the numerical results from the WENO scheme which was used to evaluate the breaking solitary wave run-up will be presented and compared to experimental results.

\subsection{Wave surface elevation and velocity time histories}

The water surface time histories at eight different locations extending from the toe of the slope to locations above the position of the initial shoreline for an incident relative wave height $H / h_{0}=0.263$ breaking on a $1: 19.85$ slope are presented in figure 6 . Both the numerical results, using the WENO scheme, and the corresponding experimental results using the capacitance wave gauge are shown. (Note that for ease of comparison the scale of the ordinate of each part of the figure is the same.) It can be seen that as the wave runs up the slope its height increases gradually and the front face steepens due to nonlinear effects. At breaking, the front face becomes vertical, and shoreward of this position the wave height decreases significantly. The numerical scheme appears to model this process of wave shoaling and decaying well, as the good agreement between the numerical and experimental results demonstrates. At locations close to the initial shoreline position, e.g. figure $6(e, f)$, generally the numerical scheme predicts a steeper front and rear water surface time history than the experiments. Since the breaking process is only represented as a sharp discontinuity in the numerical model, these differences are probably due to the over-simplified numerical modelling of the wave breaking process. In addition, where wave breaking is accompanied by a plunging jet with its associated air entrainment the accuracy of the measurements may be somewhat less.

Zhang (1996) showed, by numerical simulation, that dispersive effects may be important during the run-down process for non-breaking solitary wave run-up. This also may be true for breaking solitary waves, where in figure $6(b, c, d)$ at a nondimensional time of approximately $t^{*}=60$ to 70 it is seen that the numerical scheme is relatively poor in treating run-down. 

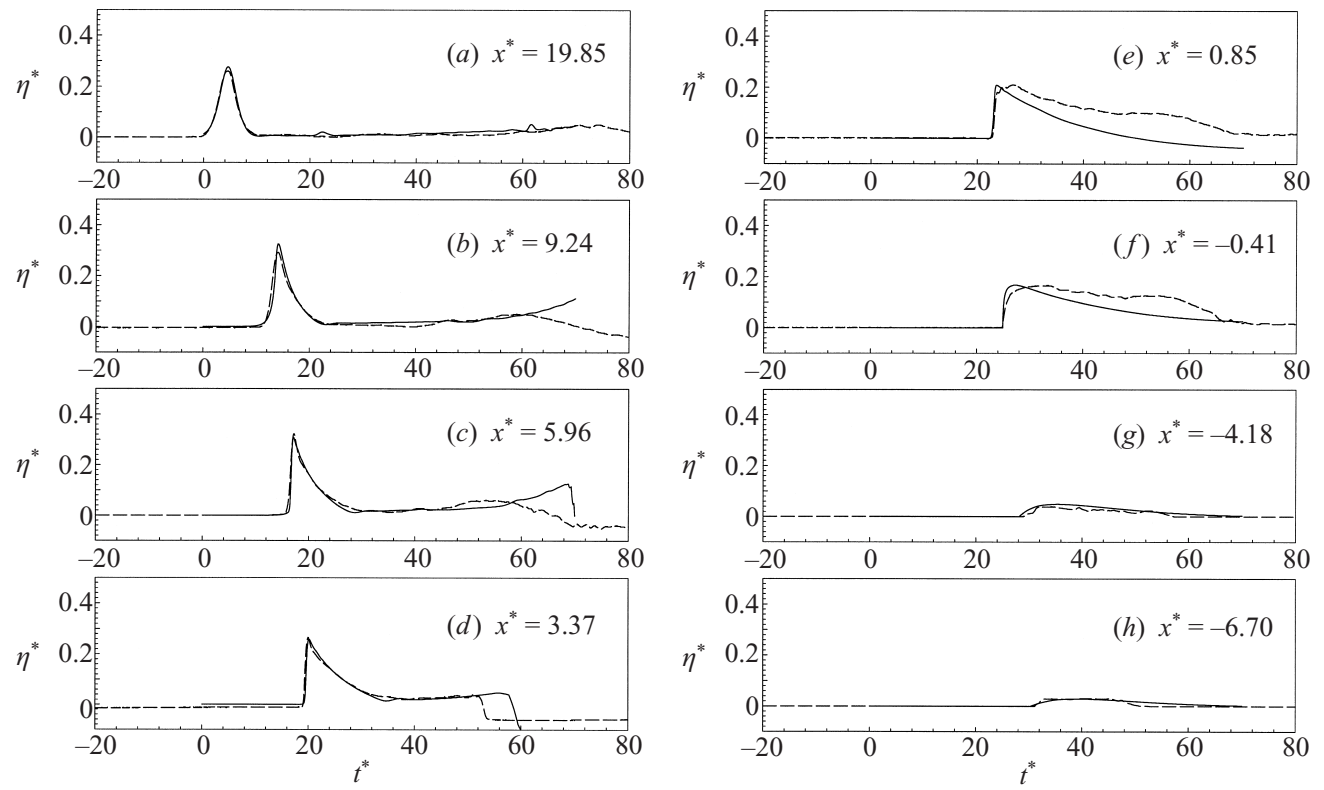

FIGURE 6. $(a-h)$ Run-up of a solitary wave with $H / h_{0}=0.263$ on $1: 19.85$ slope. Normalized water surface time histories are shown as a function of normalized time at different locations. The solid line corresponds to the numerical simulation, the dashed line corresponds to our experimental data (CIT) from the capacitance wave gauge.

The reflected wave associated with solitary wave run-up mentioned in the literature usually refers to the wave that arises both from the wave that reflects from the slope before the wave reaches its maximum run-up and the wave generated by the run-down process. As the wave is in the process of running up the slope a reflected wave is caused by the interaction of the wave and the slope. This is difficult to discern in figure 6, but it has been shown to occur by $\mathrm{Li}(2000)$ where detailed experiments were conducted to isolate this effect. In these experiments a $1: 15$ slope was used which extended only from the tank bottom to the initial shoreline position, with a collection box shoreward of and connected to the end of the slope. Thus, when the incident wave ran up the slope, the portion of the wave that would normally comprise the run-up tongue, and hence the run-down tongue, flowed over the shoreward end of the slope and was captured in the collection box. The height of this reflected wave due only to the incident wave-slope interaction was found to be of the order of $10 \%$ of the incident wave height. For the complete slope discussed herein the run-down tongue that forms after the time of maximum run-up (referring in advance to figure $8 g$ ) runs down the slope causing a hydraulic jump near the position of the initial shoreline as shown at $x^{*} \approx 2$ in figure 8 and in the vicinity of $x^{*}=3.37$ and $t^{*}=52$ in figure $6(d)$. The hydraulic jump does not propagate, as can be seen in figure $8(h-l)$, but it is the generating mechanism for the offshore propagating reflected wave associated with the run-down process.

Figure 7 shows normalized water particle velocity time histories during the run-up process at mid-depth and at four locations along the slope for a relative incident wave height of $H / h_{0}=0.263$. (The velocities are normalized by $\sqrt{g h_{0}}$.) The experimental conditions are the same as for the water surface time histories presented in figure 6 . For the location near the toe of the slope $\left(x^{*}=19.85\right)$, the numerical result agrees 

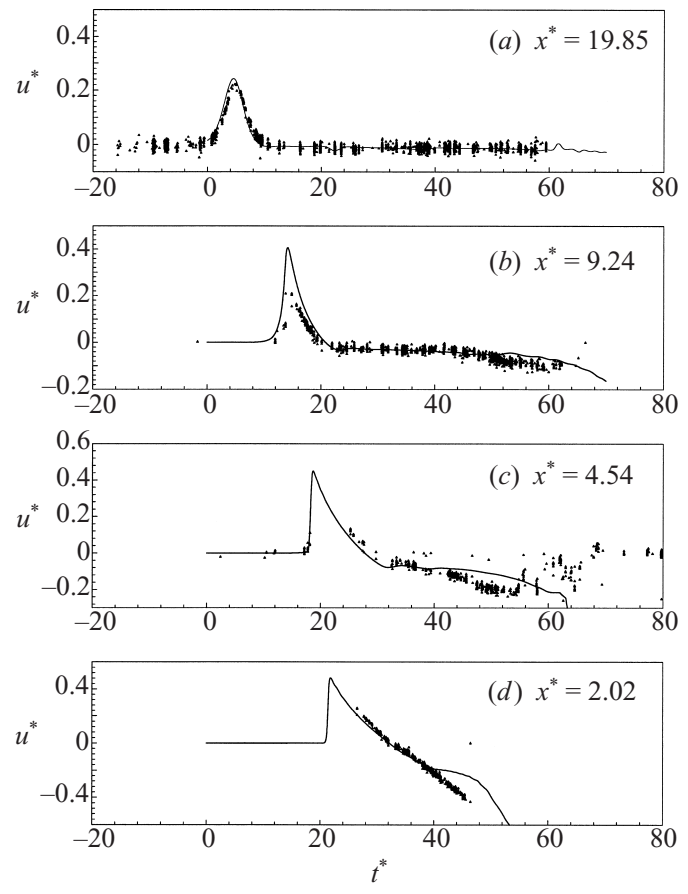

FIGURE 7. $(a-d)$ Run-up of a solitary wave with $H / h_{0}=0.263$ on $1: 19.85$ slope. Normalized water particle velocities are shown as function of normalized time at different locations. The solid line is the result of numerical simulation, the triangles are our experimental data (CIT) from LDV.

well with the experiments. The velocity time history is similar in shape to the original solitary wave, which shows that the reflection from the slope before the run-down process must indeed be small. The numerical simulation overestimates the maximum velocity near the point of wave breaking, as can be seen in figure $7(b)$. In the breaking region experimental data are not available due to the presence of bubbles generated by the plunging jet of the breaking wave which obscure and scatter the laser beam of the LDV. Overall, the numerical results agree well with the experimental results except in the region close to breaking where the details of wave breaking are so complex that they cannot be simulated by this simplified shock model.

\subsection{Free surface profiles}

The free surface profiles, i.e. the variation of the water surface elevation with distance, for an incident wave height of $H / h_{0}=0.30$ are presented in figure 8 for different non-dimensional times. Figure 8 shows both the run-up and run-down processes; $x^{*}=19.85$ is the toe of the slope since the beach slope is $1: 19.85$. The numerical results are compared to the experimental data of Synolakis (1986) attained in the CIT wave tank. As the wave shoals, the front face becomes steeper than the rear face and the wave shape becomes asymmetrical, see figure $8(b, c)$. The numerical results clearly show this trend and are reasonably well confirmed by the experimental data. At $t^{*}=10$ the numerical results overestimate the maximum height of the wave by about $10 \%$. As time increases, the front face becomes steeper and ultimately is vertical. The position at which this occurs is defined as the breaking point in the numerical model. It occurs for $15<t^{*}<20$. The wave height reaches a maximum value at this time and decreases after the wave breaks. As described before, the wave probe cannot 

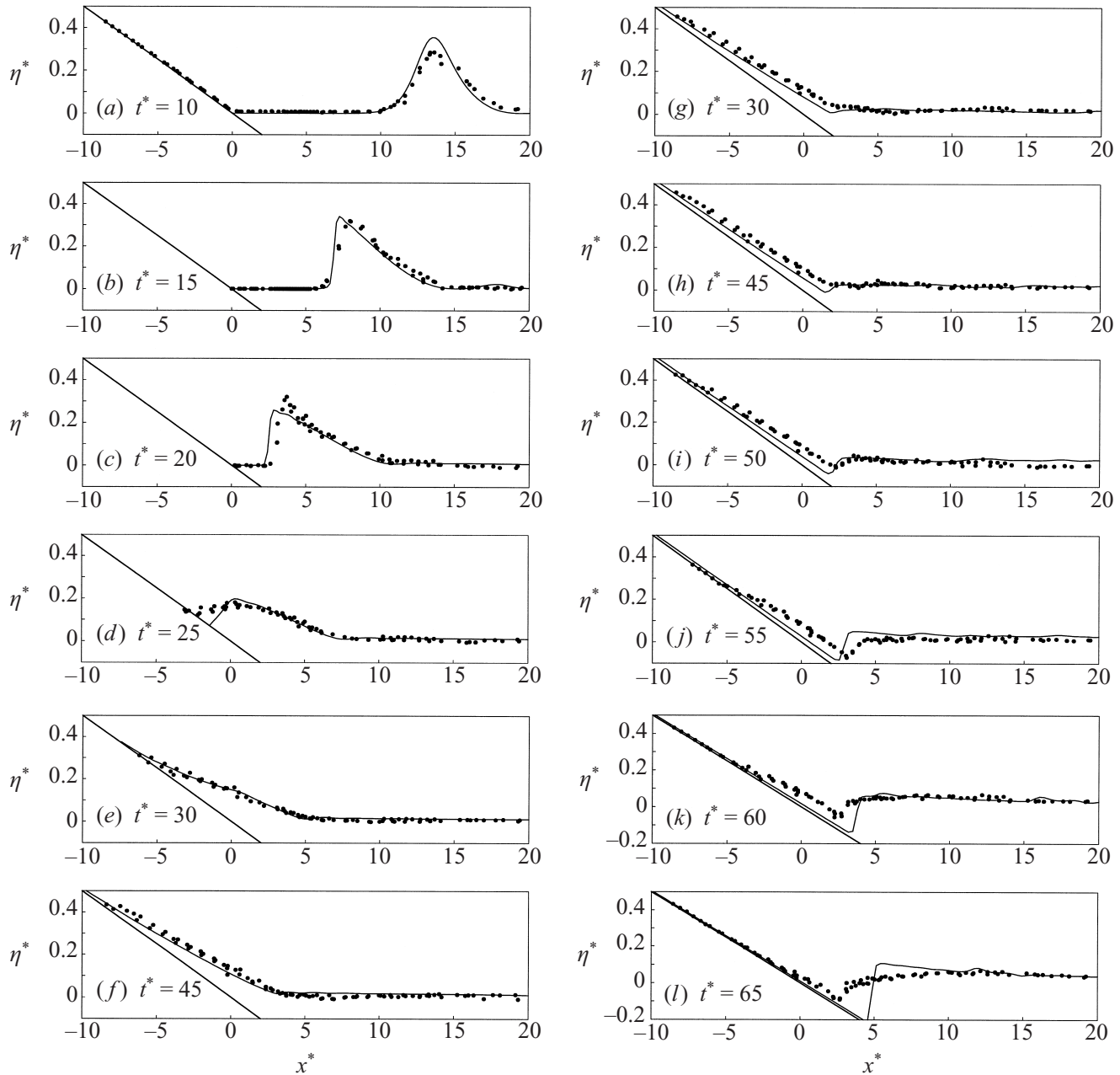

FIGURE 8. $(a-d)$ Run-up of a solitary wave with $H / h_{0}=0.3$ on $1: 19.85$ slope. Normalized water surface profiles are shown as a function of normalized distance from the initial shoreline at different times. The solid line is the result of numerical simulation, the black circles are the experimental data from Synolakis (1986).

accurately measure the details of the front of the breaking wave because of the air entrained during breaking. Nevertheless, the shock-like front face of the breaking wave is seen in figure $8(b, c)$. There appears to be a difference in the location of the front face of the breaking wave between the numerical data and experimental data as seen in figure $8(\mathrm{c})$. Considering the violence of the wave breaking process and the simplicity of the numerical model, this difference may either be caused by the over-simplified model or the measurement error associated with the breaking wave or a combination of both.

As the breaking wave propagates up the slope, it collapses near the initial shoreline position and the wave height decreases dramatically. However, away from the front tip of the wave, the shape changes slowly. The physical length of the wave collapse region is of the order of the initial water depth, which is small compared to the characteristic length of the solitary wave. This implies that, on including the shock structure into 
the long wave model, the long wave assumptions should still be valid during most of the wave breaking process. The favourable agreement between the numerical and experimental results found in figure $8(d-f)$ provides additional support for the shock model. There are some differences between the numerical and experimental data near the run-up tip as can be seen in figure $8(g, h)$. The differences in the region after breaking and in the vicinity of the run-up tip may be due to at least two features of the analytic treatment: (i) the assumption of the hydrostatic pressure distribution used in the shallow water wave equations may not apply in the breaking or post breaking region, as explained by Lin et al. (1999), and (ii) the effect of bottom friction is not included in the numerical model used in this study. The latter might be the most important cause of the observed differences between the numerical and experimental results.

The wave run-down process begins after the wave reaches its maximum run-up position $\left(\right.$ at $\left.t^{*}=42\right)$. At the beginning of this process the water retreats as the trailing edge of the solitary wave continues to propagate toward the slope. This retreating flow interacts with the wave tail and creates a region of large free surface curvature near the initial shoreline position, developing a 'hydraulic jump' near the initial shoreline as seen in figure $8(j)$. The hydraulic jump is modelled as a discontinuity (a shock) in the numerical method also. The abrupt angle of the water surface obtained in the numerical results shown in figure $8(k, l)$ is not realistic since the NLSW assumption that the horizontal velocity is constant with depth, i.e. negligible vertical acceleration, is no longer valid in this region. In the laboratory the front collapses and air entrainment occurs here. However, both the position of the jump and the height difference across the jump are predicted fairly well by the numerical method.

\subsection{Shoreline movement and maximum run-up}

The variation of the shoreline position, i.e. the normalized run-up, as a function of normalized time is presented in figure 9 for a solitary wave with $H / h_{0}=0.30$ incident on a $1: 19.85$ slope. The experimental data were obtained using a high-speed video camera mounted above the slope with the focal plane parallel to the slope. As the wave propagates up the slope, the contact line between the tip of the run-up tongue and the dry bed is used to locate the shoreline at different times using the scale attached to the surface of the slope. Because of the limited distance that the high-speed video can cover on the slope, the experiment was repeated six times with the high-speed video camera moved to a new location each time. In this way the run-up time history was recorded up to the maximum run-up. The results from different runs were assembled, and the discontinuities noted among these sets of data may be attributed to this experimental procedure. During the run-down process, since the surface of the slope was already wet, the contact line was indistinct and almost undetectable in the video recording. Thus, only the run-up phase of the shoreline position is presented. Good agreement was found between the numerical results and experiments, which shows that the WENO numerical scheme can simulate the run-up and the shoreline position with relatively high accuracy.

The normalized maximum run-up, $R / h_{0}$, is shown as a function of incident wave height, $H / h_{0}$, in figure 10,11 and 12 for three slopes: $1: 5.67,1: 15$, and $1: 19.85$ respectively. The experimental data for the $1: 5.67$ slope are from Hall \& Watts (1953), where the solitary wave was generated by the simple impulsive motion of a vertical bulkhead. The experimental data for the $1: 19.85$ slope are from Synolakis (1986) for a range of water depths from $6.25 \mathrm{~cm}$ to $38.32 \mathrm{~cm}$. The data for $1: 15$ slope were measured by the present authors for the different water depths shown. The wave 


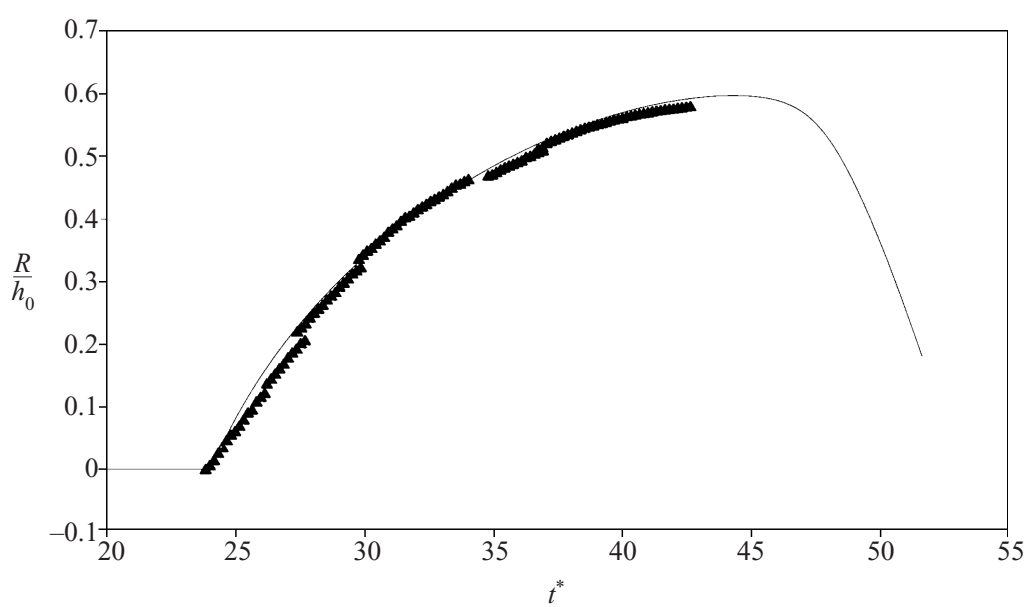

FIGURE 9. Run-up of a solitary wave with $H / h_{0}=0.30$ on $1: 19.85$ slope. The normalized shoreline position is shown as a function of normalized time. The solid line is the numerical results, the circles are experimental data from high-speed video.

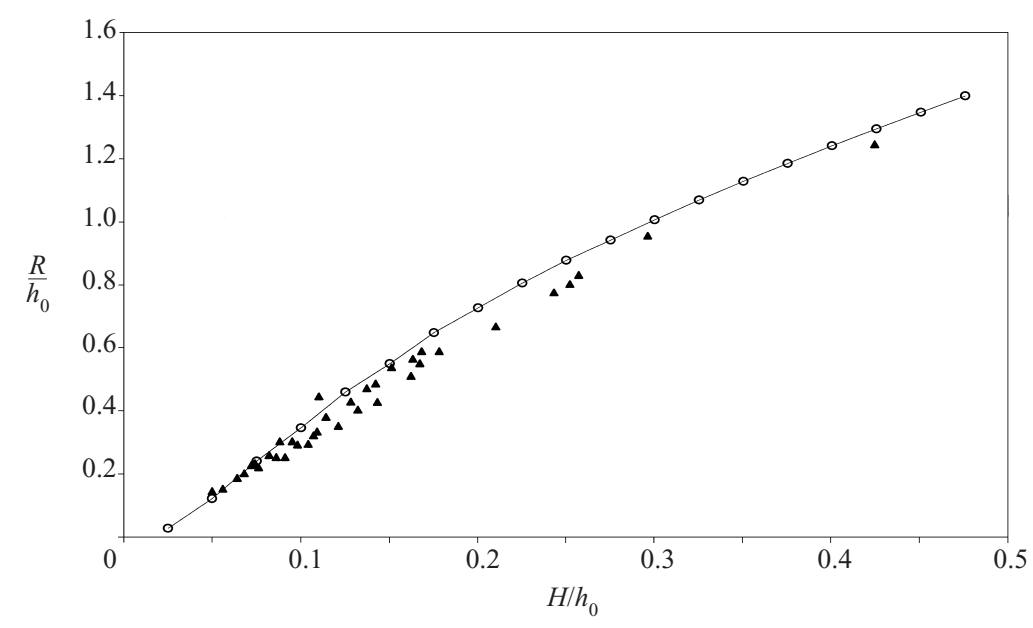

FigURE 10. Maximum run-up of a solitary wave on $1: 5.67$ slope. The normalized maximum run-up is shown as a function of incident wave height. The solid line with the hollow circles are the numerical results, the triangles are experimental data from Hall \& Watts $(1953) .\left(H / h_{o}\right)_{\text {breaking }}=0.119$.

generation system is the same for Synolakis's (1986) data and ours. The breaking condition from Synolakis (1986), i.e. $H / h_{o}=0.818(\cot \beta)^{-10 / 9}$, is used to define the non-breaking region and is shown in the captions of figure 10, 11 and 12. It should be noted that in the non-breaking region the analytical results from Synolakis (1986) and from Li \& Raichlen (2001) are in agreement with the limited data shown. These figures show clearly that the maximum run-up is predicted well by the WENO scheme combined with the nonlinear shallow wave equations.

The relatively good agreement between the numerical results of the WENO scheme and the experimental results should be investigated further, since the bore structure used to model the wave breaking process in the numerical model considerably simplifies the physical process it represents. One possible reason for the good agreement when using this simplified model may be the relatively large length scales of both 


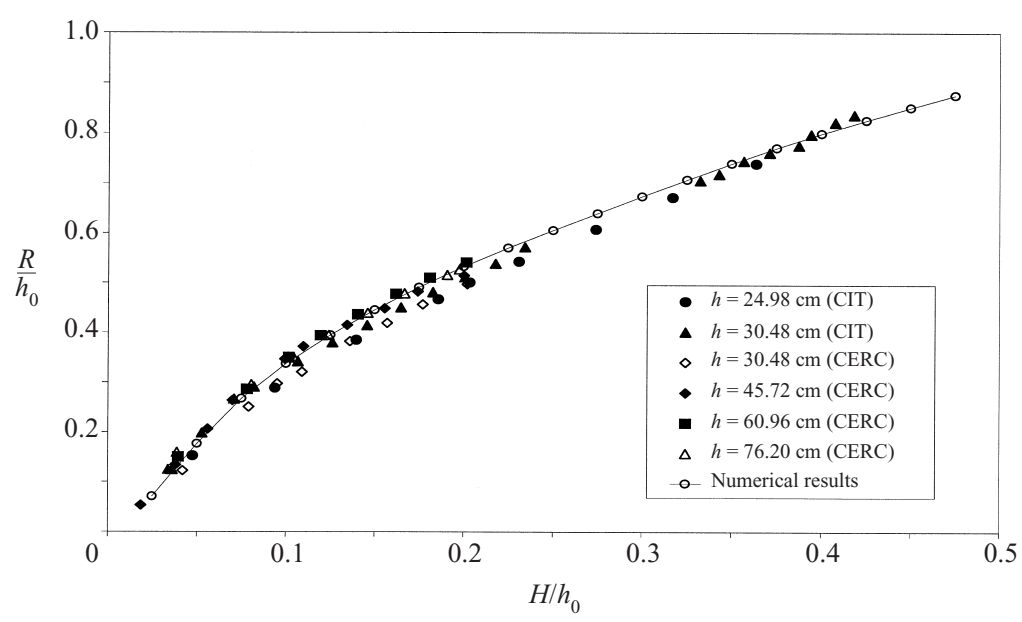

FIGURE 11. Maximum run-up of a solitary wave on $1: 15$ slope. The normalized maximum run-up is shown as a function of incident wave height. The solid line with the hollow circles are the numerical results, the symbols are experimental data. $\left(H / h_{o}\right)_{\text {breaking }}=0.040$.

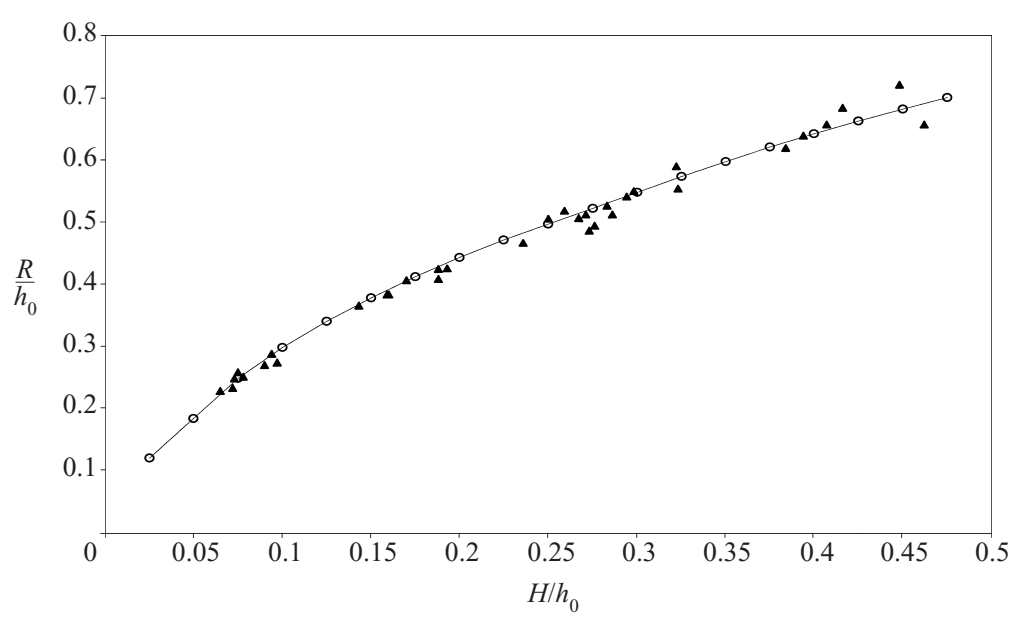

FIGURE 12. Maximum run-up of a solitary wave on $1: 19.85$ slope. The normalized maximum run-up is shown as a function of incident wave height. The solid line with the hollow circles are the numerical results, the triangles are experimental data from Synolakis $(1986)$. $\left(H / h_{o}\right)_{\text {breaking }}=0.030$.

the incident wave and the run-up process compared to the length scale of the wave breaking region. In other words, although the wave breaking process is complex and difficult to fully understand, the region that it affects is actually small (of the order of the water depth). Thus, from this 'macroscopic view', the wave breaking can be regarded as a sharp discontinuity or an energy sink. In addition, the equations defining the numerical model correctly represent mass and momentum conservation across the breaking wave. If the mass and momentum exchange across the discontinuity are treated correctly, then, like the hydraulic jump or the dam-break problem, one should be able to obtain the global parameters of the characteristics of the wave run-up process such as the maximum run-up and the wave amplitude time histories with reasonable confidence.

The normalized maximum run-up, $R / h_{0}$, is shown as a function of the cotangent 


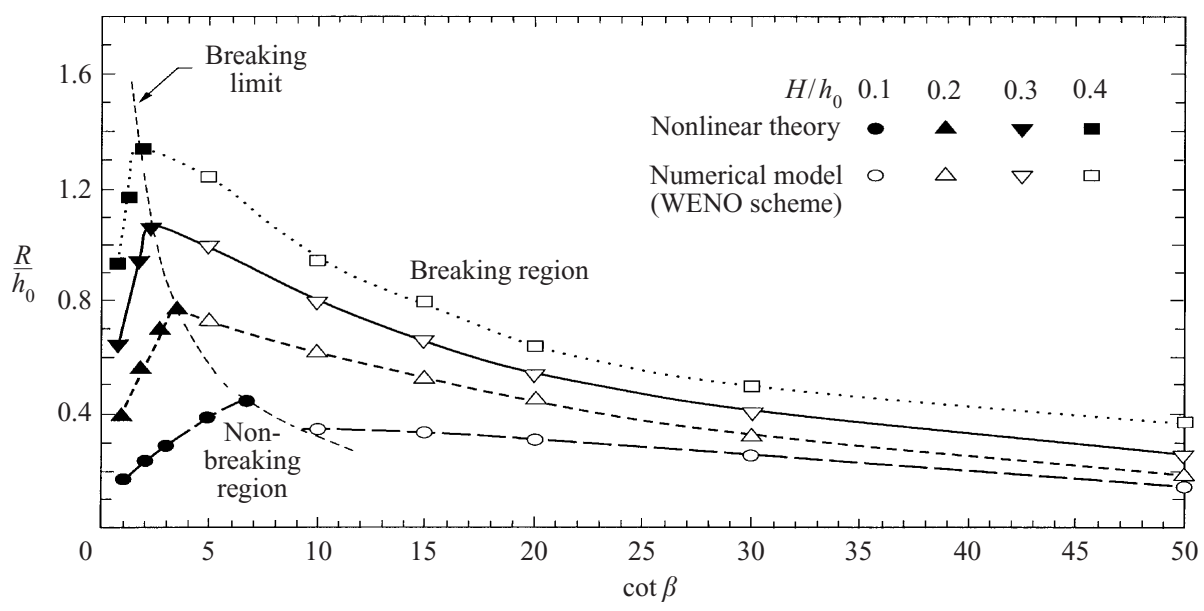

FIGURE 13. Maximum run-up of a solitary wave as a function of slope angle for non-breaking and breaking waves for several incident relative wave heights.

of the angle of the slope, $\cot \beta$, in figure 13. Only the numerical results for both non-breaking and breaking solitary wave run-up are presented with curves drawn through these computed points. (These data have been confirmed experimentally up to $\cot \beta \approx 20$ as seen in the agreement between experiments and computation-figures 10 , 11 and 12.) The dash-dotted line shown separates non-breaking from breaking wave run-up; this has been evaluated using the analysis presented by Synolakis (1986). (The maximum run-up for non-breaking solitary waves was calculated from the nonlinear theory of Li \& Raichlen (2001), and that for breaking solitary wave was obtained from the WENO numerical model described herein.) It can be seen in figure 13 that the variation of the maximum run-up with the angle of the slope relative to horizontal is different for non-breaking solitary waves and breaking solitary waves. In the non-breaking region, the maximum run-up increases as the angle of the slope decreases, while for breaking waves the maximum run-up decreases as the slope becomes gentler. These trends of run-up with beachslope for non-breaking and breaking waves have been noted before, e.g. by Hunt (1959) for periodic waves. For non-breaking solitary waves, Synolakis (1987) developed an expression for maximum run-up that increases as the angle of the slope decreases for the same offshore relative wave height. Kobayashi \& Karjadi (1994) developed a surf-similarity parameter to describe breaking solitary wave run-up that was proportional to $\tan \beta$, leading to the opposite condition compared to non-breaking solitary waves, i.e. as the slope decreases the run-up decreases for the same offshore wave characteristics. It is postulated that one effect of slope on controlling run-up for breaking solitary waves is related to the energy dissipation associated with wave breaking (a significant fraction of the incident wave energy). This can be seen in the following argument. As the angle of the slope decreases for a given offshore wave height and depth, the wave will break further offshore. If one uses the bore model to describe the energy dissipated during the breaking and post-breaking process, as the angle of the slope decreases the bore will propagate further along the slope leading to an increase in the total energy dissipated. This explains, to some extent, the observation that for breaking waves with the same offshore conditions, as the angle of the slope decreases the run-up also decreases. 


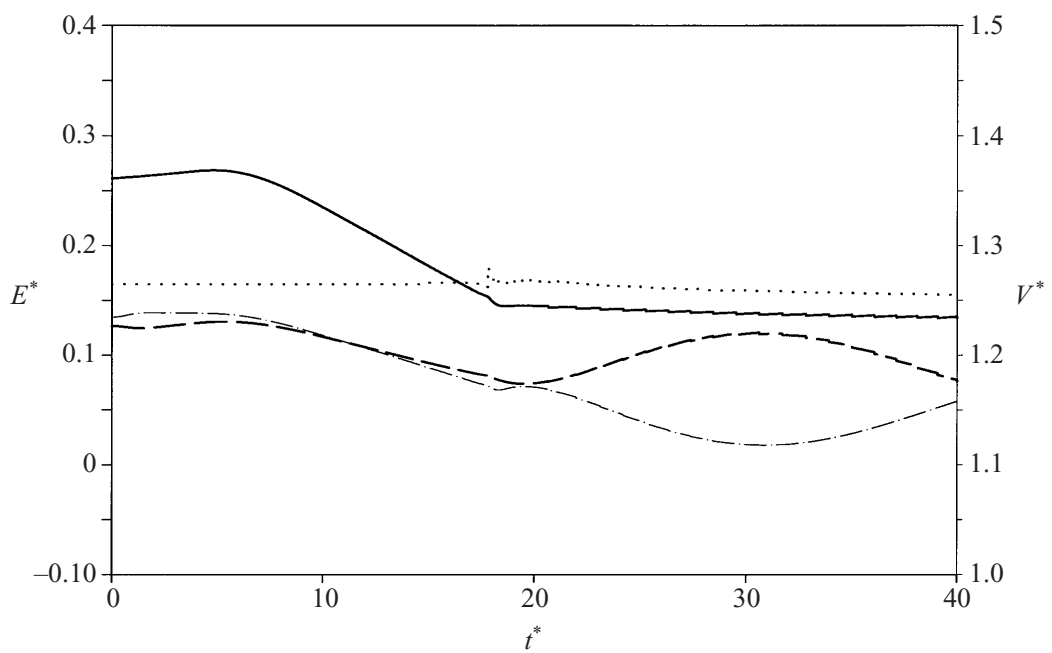

FIGURE 14. Calculated normalized energy of breaking solitary run-up with $H / h_{0}=0.30$ on $1: 15$ slope as a function of normalized time. The solid line is the total energy associated with the wave, the dashed line is the potential energy, the dash-dotted line is the kinetic energy, and the dotted line is the volume of the wave.

\subsection{Energy dissipation}

It has been shown that the energy dissipation associated with the wave breaking process limits the run-up for relatively gentle slopes. The agreement between the numerical results and the experimental results for wave shape and water surface time histories during run-up as well as the maximum run-up suggests that the shock simplification used in the numerical model can represent, to some extent, the effects of wave breaking, at least with regard to the global properties of the wave. Thus, taking a bore, i.e. a moving hydraulic jump, as a model of the broken wave, we can estimate the energy dissipation associated with wave breaking or at least for the shoreward propagation of the broken wave. The wave breaking processes is extremely complicated. Therefore, this approach to determine the energy dissipation associated with breaking in terms of dissipation related to a bore is indeed a simplification of the process, which neglects the details of the breaking wave such as the localized energy dissipation associated with the plunging jet.

The mass conservation and momentum conservation equations for a bore have been analysed by Stoker (1957). It was found that energy is not conserved if a bore exists in the flow domain, and energy must be dissipated by the turbulence produced at the front of the bore. The WENO scheme presented here solves the mass and momentum conservation equations across the shock; the energy conservation equation is automatically solved according to the above analysis. If one calculates the total energy in the domain directly by integrating the potential energy and kinetic energy throughout the domain used for computation an estimation of the total energy dissipated by wave breaking can be obtained based on the bore-breaking wave simulation.

Figure 14 shows the time history of the energy and volume of a solitary wave with wave height $H / h_{0}=0.30$ as it runs up a $1: 15$ slope. The energy and volume were normalized as in (2.38). The total energy is approximately conserved before wave breaking. (A small increase in total energy during the initial stage of calculation can be seen in figure 14; it needs further investigation.) When the wave breaking process 


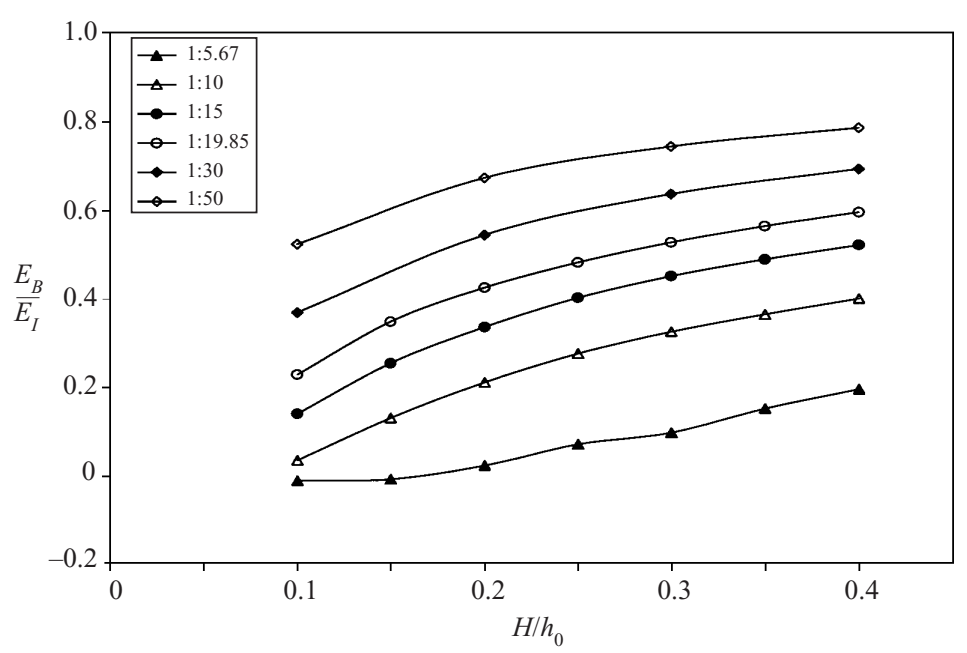

FIGURE 15. Ratio of dissipated energy to the incident wave energy for breaking solitary wave run-up as a function of relative incident wave height for various slopes. Numerical results.

begins, both the kinetic energy and the potential energy decrease with time. This energy dissipation process appears to end when the wave reaches the initial shoreline position and the run-up process begins $\left(t^{*} \approx 18\right)$. The total energy is conserved as the kinetic energy decreases and potential energy increases and the run-up and run-down proceeds. The wave reaches the maximum run-up position around $t^{*}=31$, and the potential energy reaches a maximum. The kinetic energy at this position is very small, as seen in figure 14. This small amount of energy may be associated with the mild reflected wave from the slope or the small and negligible water particle velocity associated with the run-up tongue. Past this point, the potential energy decreases and the kinetic energy increases as the water begins to run down the slope. The variation with time of the total volume associated with the wave is also presented in figure 14 . The volume is defined as the integral with distance of the deviation of the water surface from the at-rest water surface at each non-dimensional time, $t^{*}$. It is seen that the volume is constant during the run-up and run-down process, which shows that mass is conserved during computation, with slight variations near the time at which the wave reaches the original shoreline.

The total energy dissipation for the breaking solitary wave run-up can be estimated by comparing the total energy at the maximum run-up position, i.e. $t^{*}=31$ in figure 14, to the total energy in the incident wave. This is presented in figure 15 where the ratio of the dissipated energy due to breaking, $E_{B}$, to the incident wave energy, $E_{I}$, is shown as a function of the incident wave height, $H / h_{0}$, for six slopes varying from $1: 5.67$ to $1: 50$. It can be seen that as the incident wave height increases, the portion of the incident energy which is dissipated increases. For the $1: 5.67$ slope, wave breaking first occurs when $H / h_{0}=0.119$ according to the breaking criterion proposed by Synolakis (1986). The numerical results confirm this in that the energy dissipation is almost zero for $H / h_{0} \leqslant 0.15$. The energy dissipation on more gentle slopes is larger than that on the steep slope for the same incident wave height for the reasons discussed earlier. For example, almost $40 \%$ of the incident energy will be dissipated on a $1: 15$ slope for an incident solitary wave with $H / h_{0}=0.30$, but only $5 \%$ on $1: 5.67$ slope. 


\section{Conclusion}

The following main conclusions were drawn based on the experimental and numerical results:

(a) The numerical method developed in this study to predict the run-up of breaking waves provides a somewhat simple but reasonably good prediction of various aspects of the run-up process. The results agree well with the experimental data for breaking solitary waves.

(b) The numerical method is stable, simple to implement and requires relatively small computational resources.

(c) The numerical results for the 'global parameters' of solitary wave run-up such as the maximum run-up and the wave profile agree reasonably well with the experiments for both wave breaking conditions, i.e. wave breaking with and without rigorous splash-up.

(d) The detailed characteristics of the wave breaking process, such as the shape of plunging jet and the subsequent splash-up, cannot be described by the numerical model.

This investigation was supported by the National Science Foundation through Grant No. CMS-9523414 for which the authors are grateful. The authors appreciate the detailed reviews of the referees which has led to significant improvement in the revised manuscript.

\section{REFERENCES}

Brennen, C. \& Whitney, A. K. 1970 Unsteady free surface flows; solutions employing the Lagrangian description of the motion. Proc. 8th Symp. Naval Hydrodynamics, August 24-28, pp. 117-145. Office of Naval Research and National Research Council of National Academy of Science.

DodD, N. 1998 Numerical model of wave run-up, overtopping, and regeneration. J. Waterway, Port, Coastal, Ocean Engng ASCE 124, 73-81.

Goring, D. G. 1979 Tsunami: the propagation of long waves onto a shelf. PhD Thesis, California Institute of Technology.

Grilli, S. T., Svendsen, I. A. \& Subramanya, R. 1997 Breaking criterion and characteristics for solitary waves on slope. J. Waterway, Port, Coastal, Ocean Engng ASCE 123, 102-112.

Hall, J. V. \& WaTts, J. W. 1953 Laboratory investigation of the vertical rise of solitary waves on impermeable slopes. Beach Erosion Board, US Army Corps of Engineering, 33.

Harten, A., Engquist, B., Osher, S. \& Chakravarthy, S. 1987 Uniformly high order essentially non-oscillatory schemes. J. Comput. Phys. 71, 231-303.

Hibbert, S. \& Peregrine, D. H. 1979 Surf and runup on a beach: a uniform bore. J. Fluid Mech. 95, 323-345.

Hunt, I. A. 1959 Design of seawalls and breakingwaters. J. Waterway and Harbor Div. ASCE 85, $123-152$.

JIANG, G. \& SHU, C.-W. 1996 Efficient implementation of weighted ENO schemes. J. Comput. Phys. 126, 202-228.

Kawata, Y., Benson, B. C., Borrero, J. C., Davies, H. L., De Lange, W., Imamura, F., Letz, H., NotT, J. \& Synolakis, C. E. 1999 Tsunami in Papua New Guinea was as intense as first thought. EOS Trans. AGU 80, No. 9, March 2, 1999.

KobaYASHI, N. \& KARJADI, E. A. 1994 Surf-similarity parameter for breaking solitary wave run-up. J. Waterway, Port, Coastal, Ocean Engng ASCE 120, 645-650.

LI, Y. 2000 Tsunamis: Non-breaking and breaking solitary wave run-up. Rep. KH-R-60, W. M. Keck Laboratory of Hydraulics and Water Resources, California Institute of Technology, Pasadena, CA.

Li, Y. \& Raichlen, F. 2001 Solitary wave run-up on plane slopes. J. Waterway, Port, Coastal Ocean Engng ASCE 127, 33-44. 
Lin, P., Chang, K.-A. \& LiU, P. L.-F. 1999 Runup and run-down of solitary waves on sloping beaches. J. Waterway, Port, Coastal, Ocean Engng ASCE 125, 247-255.

LiU, X.-D., Osher, S. \& Chan, T. 1994 Weighted essentially non-oscillatory schemes. J. Comput. Phys. 115, 200-212.

SHU, C.-W. 1998 Essentially non-oscillatory and weighted essentially non-oscillatory schemes for hyperbolic conservation laws. In Advanced Numerical Approximation of Nonlinear Hyperbolic Equations (ed. A. Quarteroni), pp. 329-432. Springer.

Stoker, J. J. 1957 Water Waves. Interscience Publishers.

Synolakis, C. E. 1986 The run-up of long waves. PhD Thesis, California Institute of Technology, Pasadena, CA.

Synolakis, C. E. 1987 The run-up of solitary waves. J. Fluid Mech. 185, 523-545.

Titov, V. V. \& Synolakis, C. E. 1995 Modeling of breaking and non-breaking long-wave evolution and run-up using VTCS-2. J. Waterway, Port, Coastal, Ocean Engng ASCE 121, 308-461.

Wu, T. Y. 1979 Tsunamis. Proc. Natl Science Foundation Workshop, Pasadena, CA, pp. 110-149.

YANG, J. Y. \& Hsu, C. A. 1993 Computations of free-surface flows: 2-dimensional unsteady bore diffraction. J. Hydraul. Res. IAHR 31, 403-414.

ZELT, J. A. 1991 The run-up of non-breaking and breaking solitary waves. Coastal Engng 15, 205-246.

Zelt, J. A. \& Raichlen, F. 1990 A Lagrangian model for water-induced harbor oscillations. J. Fluid Mech. 213, 203-225.

Zelt, J. A. \& Raichlen, F. 1991 Overland flow from solitary waves. J. Waterway, Port, Coastal, Ocean Engng ASCE 117, 247-263.

Zhang, J. E. 1996 Run-up of ocean waves on beaches. PhD Thesis, California Institute of Technology, Pasadena, CA. 